\title{
STUDIES ON THE ELASTIC PROPERTIES OF HUMAN ISOLATED AORTA
}

\author{
By PHILLIP HALLOCK AND IKEL C. BENSON \\ (From the Department of Medicine and Graduate School of Medicine, University of \\ Minnesota, Minneapolis)
}

(Received for publication February 11, 1937)

THE VOLUME-ELASTICITY RELATIONS OF THE AORTA TO PRESSURE AND AGE

For the most part the study of the physiology of circulation has been confined to the heart and to a somewhat lesser extent to the peripheral blood vessels. The importance of the large central vessels as a functional unit of the cardiovascular apparatus has not been fully appreciated, chiefly because they have been looked upon merely as channels for the passage of blood from the heart to the periphery. But their relationship to the cardiovascular apparatus extends beyond this conception. By virtue of its inherent elastic properties, the aorta and its main branches become an essential functional unit of the circulatory system and contribute to an appreciable extent in maintaining the efficiency of the circulation. We have sought in this present investigation to study the elasticity characteristics of isolated human aortas with a view of determining the effect of these responses to the work of the heart and, further, to obtain quantitative data regarding the readjustments that may occur in the aorta under conditions of hypertension and arteriosclerosis.

Roy (1) in 1880, studied the relation between the internal pressure and the volumetric capacity of arteries by making direct physical measurements on isolated aortas of animals. By means of a series of curves, he showed that the aortic walls were most extensible at pressures corresponding (approximately) to their normal blood pressures, and that at higher pressures the extensibility of the wall of the vessel was considerably impaired. In making his observations, he allowed 20 to 30 minutes for the intra-arterial pressure to rise from 0 to $200 \mathrm{~mm}$. $\mathrm{Hg}$. The reason for making his observation slowly, was to reduce to a minimum any influence due to elastic "after-action"-the property of elastic tissue such as arteries to continue expanding for some time if the pressure or tension be constantly main- tained. Bramwell and Hill (2), in 1922, after measuring the slopes at various points on Roy's curves, calculated the velocity of the pulse wave at various pressures. They found that the velocities, at pressures of $80 \mathrm{~mm}$. $\mathrm{Hg}$ (equivalent to normal diastolic pressure in man), obtained from Roy's work were lower than those observed in man. Bramwell and Hill (2) in discussing Roy's method, stated, " From a point of view of static effect of the diastolic pressure of the arteries, he (Roy) succeeded; from that, however, of the dynamic effects occurring in a rapid cycle of events associated with the pulse, his precautions aggravated the error and must have caused the increase of volume per millimeter of mercury to be much larger than that occurring in a rapid change of pressure." They believed, therefore, that the velocities calculated from Roy's work were low because of the effect of elastic " afteraction." To eliminate the possibility of elastic "after-action," these investigators devised a means of measuring the pulse wave velocity directly on isolated arteries (common carotid), and their results compared quite favorably with those observed in man. The vessels used in their study showed no microscopic postmortem evidence of arterial disease. Bramwell, Downing and Hill (3), in an attempt to arrive indirectly at a qualitative picture of arterial rigidity by numerical integration, constructed curves from their data relating total volume of an artery to the pressure inside it. They abandoned Roy's method because it did not measure the sharp momentary increase of volume such as follows the heart beat. They obtained their mean normal curve from a set of curves representing six normal individuals in the ages ranging from 8 to 45 years.

In this study, in order to obtain characteristic curves for the various age groups instead of one curve for the combined ages, the volume-elasticity characteristics of eighteen aortas were experi- 


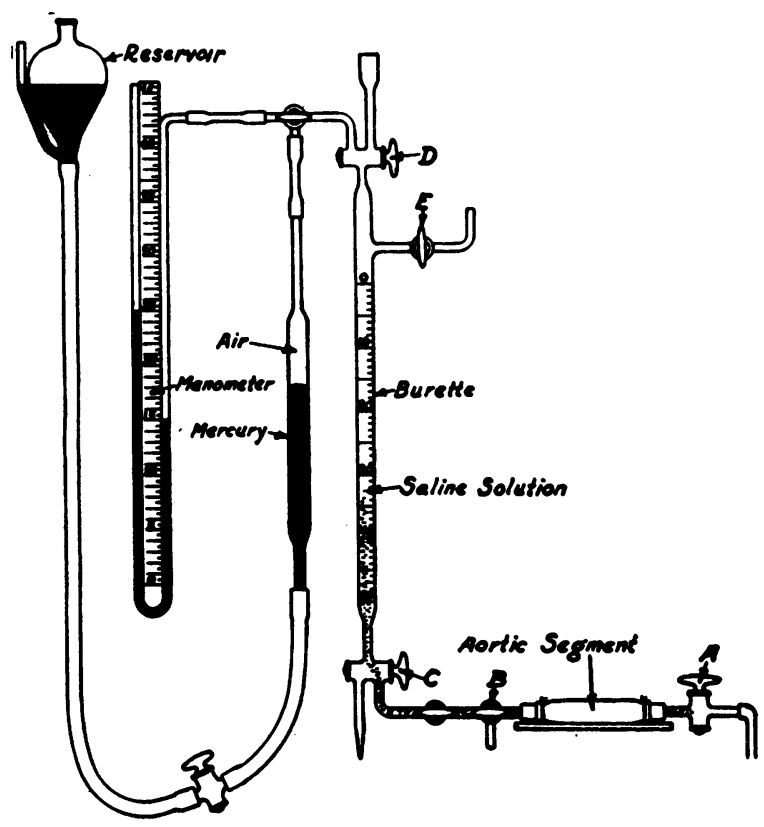

Fig. 1. Apparatus for Determining Increase in Size of Segment of Thoracic Aorta under IncreasING PRessure

The mounting of the apparatus is not shown in this figure.

mentally obtained and plotted for the various age groups. The observations were usually made on the same day that the autopsy was performed. Only aortas of the normal type, as revealed by gross postmortem examination, were accepted for use in this study. In no instance was any aorta accepted for study where its heart showed any gross pathological changes, such as hypertrophy, dilatation, valvular defects or coronary disease with the exception of those aortas isolated for study for age group from 71 to 78 years. In the hearts of those in this period of life, some degree of coronary disease is to be expected, but the changes noted were indeed minimal considering the age.

The blood pressure readings noted from the clinical records were all within normal limits, i.e. between 110 and $160 \mathrm{~mm}$. $\mathrm{Hg}$ systolic and between 60 and $95 \mathrm{~mm}$. $\mathrm{Hg}$ diastolic pressure.

The volume-elasticity characteristics were all obtained by direct physical measurements from isolated segments of the thoracic aorta measuring from 8 to $10 \mathrm{~cm}$. in length. For obtaining these physical measurements, the apparatus shown in Figure 1 was designed so that the volume and pressure readings could be obtained very quickly. The segment of aorta, to be used in the experiment, was taken from the descending aorta just beyond the arch. It was necessary to strip off practically all of the dense fibrous tissue constituting the adventitia in order to ligate the intercostal vessels tightly. While the aorta was being prepared for this experiment it was kept constantly moistened with normal saline solution. Then the segment of aorta was attached to the apparatus as shown in Figure 1. The aorta was filled with normal saline solution from the burette, avoiding any air bubbles in the aorta or the connected parts, at a pressure equal to that of a column of $10 \mathrm{~cm}$. of normal saline solution which is approximately equal to $7.3 \mathrm{~mm}$. $\mathrm{Hg}$. This small amount of pressure was necessary to keep the aorta in its normally distended condition. The amount of normal saline solution in the system from Stopcock $B$ to $A$ was measured by allowing it to pass through $A$ into a beaker and weighing the solution in the beaker accurately. The cubic contents of the glass parts having been previously determined, it was a simple calculation to determine the cubic contents of the aortic segment at the given pressure or its volume at approximately $7.3 \mathrm{~mm}$. $\mathrm{Hg}$ pressure. The aortic segment was filled again under $7.3 \mathrm{~mm}$. $\mathrm{Hg}$ pressure. Stopcocks $A$ and $C$ were then closed and the burette filled to the zero mark with normal saline solution. Stopcock $C$ was next opened thus subjecting the segment of the aorta to the hydrostatic pressure of the height of the column of saline solution in the burette only and the reading on the burette recorded as the first reading. This reading varied according to the extensibility of the particular aorta and generally amounted to values approximating 25 $\mathrm{mm}$. $\mathrm{Hg}$ pressure. Stopcock $E$ was then closed and $D$ opened so as to connect the burette with the air and mercury pressure chamber and the manometer. For the next reading, the reservoir containing mercury was raised so as to increase the pressure by approximately $25 \mathrm{~mm}$. $\mathrm{Hg}$. Immediately thereafter and at the same instant of time, the manometer was read by one observer and the burette height was read by another observer. This procedure and readings thereof were repeated at increasing steps of approximately $25 \mathrm{~mm}$. $\mathrm{Hg}$ up to about $230 \mathrm{~mm}$. $\mathrm{Hg}$ pressure. 
In calculating the pressure within the aortic segment, the pressure of the column of saline solution in the burette above the level of Stopcock $B$ was added to the mercury manometer reading and the sum of the two constituted the pressure within the aorta. The specific gravity of the saline solution was slightly over 1.0 while that of mercury is 13.6. By means of this apparatus it was possible to reduce the effects of elastic "after-action" and thus obtain characteristic curves which would accurately represent dynamic effect of cardiac events on pressure and volume changes occurring in the aortic chamber. ${ }^{1}$ The first set of readings was checked by repeating the above procedure. In those aortas which developed leaks at high pressure, the entire experiment was rejected. The time consumed in making the nine observations of pressure and volume readings from $7.3 \mathrm{~mm}$. to $230 \mathrm{~mm}$. $\mathrm{Hg}$ was approximately two minutes.

The percentage increase in volume compared to the measured volume at $7.3 \mathrm{~mm}$. $\mathrm{Hg}$ was plotted along the ordinate axis against the corresponding pressures along the abscissa. No satisfactory observations were made on aortas of ages below 16 years because of the difficulty encountered in ligating the bases of the small intercostal arteries as they emerged from the aorta. Also, no observations were made on aortas in the age group between 55 and 65 years because of the inability to find normal aortas for this period of life in the available autopsy material. Figure 2 shows the mean volume-elasticity curves of the aortas which may be considered as characteristic or normal for the various designated age groups.

Curves $a$ and $b$ are obviously S-shaped and are characteristic of arteries which are endowed with considerable elasticity, a quality common to the vascular tubing of the younger ages. They turn sharply upward at pressures lower than normal diastolic pressures and rise rapidly with increments of pressure. They tend to become horizontal as extremely high pressures are encountered. Thus at low pressures and even at

1 The term aortic chamber is here considered as a physiological unit and includes the arteries which are readily distensible. From the anatomical point of view, it constitutes the elastic arteries, i.e., the aorta, the innominate, the subclavions and proximal parts of the common carotids.

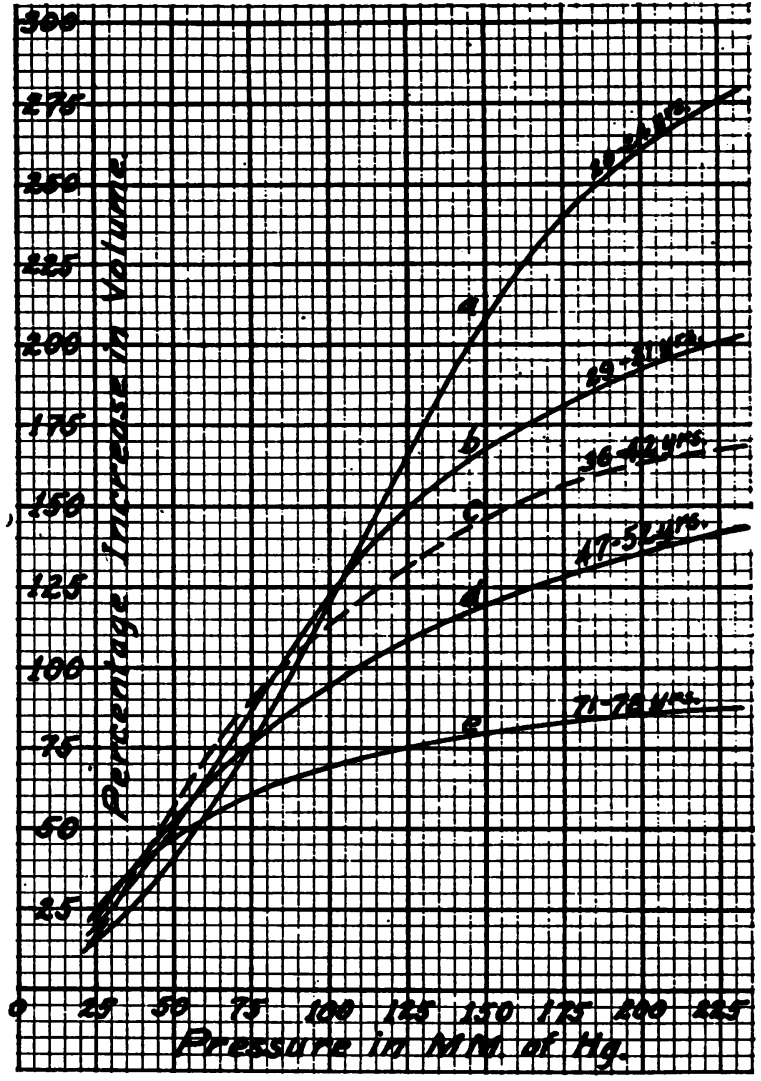

Fig. 2. Mean Volume-elasticity Curves of Thoracic Aortas at Various Age Groups

These curves show the relation of percentage increase in volume to increase in pressure for five different age groups and were constructed from the mean values obtained from a number of aortas excised at autopsy.

pressures much higher than normal diastolic pressures, the aortas belonging to these age groups do exhibit a high degree of extensibility. Only at extremely high pressures do they become relatively non-extensible. Curves $c$ and $d$ begin to show the effect of the diminution in arterial elasticity associated with advancing years. However, while considerable extensibility still exists at relatively high pressures, there is now a definite tendency for the curves to become flat, thereby exhibiting the non-elastic characteristic. Curve $e$ shows the normal effect of the ageing process on aortas. The volume now increases slightly with increasing internal pressures, and at high pressures the curve becomes almost a straight horizontal line. The histological picture of the wall of such an aorta would show it to be almost devoid of elastic tissue, as demonstrated by Zon 
(4). In addition to the lack of elastic tissue in aortas of this group, the extensibility of the vessels is further hampered by the increase of collagenous fibers (senile fibrosis) and by depositions of arteriosclerotic products in the vessel wall.

Since the aortic chamber is essentially an elastic structure as indicated by Figure 2, it follows that the aorta as well as its main branches constitute an important dynamic unit of the cardiovascular apparatus. Thus, as a result of its elastic retraction during the phase of cardiac diastole, the aortic chamber functions in the capacity of a buffer system converting a cardiac outflow which would be otherwise intermittent into one which is continuous in the capillaries.

In addition to its influence on the peripheral circulation, the aorta tends to minimize the work of the heart by facilitating the discharge of blood from the left ventricle, as pointed out by Bramwell. Such a view is indeed 'tenable when one considers the volume-elasticity curves as exhibiting the relation which exists between the.pressure in the aorta and its ability to accept the cardiac output. It will be noted from the volume-elasticity curves $a, b$, and $c$, that within the range of normal diastolic pressure only a slight elevation of pressure is required to produce a considerable increase of :volume, thus indicating the ease by which the elastic aorta can accept its ventricular outflow, providing of course other factors remain normal. But with increasing pressure, the trend of these curves is to flatten'out showing that the aortic reservoir can no longer accommodate the same volume of blood with the same small pressure increase as it formerly could. On the contrary, a much greater rise in pressure is required to receive the same output. If, for example, we consider Curve $c$, it will be noted that at a diastolic pressure of $75 \mathrm{~mm}$. $\mathrm{Hg}$ a rise of $15 \mathrm{~mm}$. of pressure will produce a 17 per cent increase in volume; while 'at $125 \mathrm{~mm}$. of pressure, a rise of $15 \mathrm{~mm}$. will produce only a 7 per cent increase of volume; and at $150 \mathrm{~mm}$. pressure, an increase of $15 \mathrm{~mm}$. will only bring about a 6 per cent increase of volume. In other words, to produce a 15 per cent increase of volume at $150 \mathrm{~mm}$. pressure, an increase of $50 \mathrm{~mm}$. would be required. This readjustment could only be brought about by an increased effort on the part of the heart in an attempt to augment. the pulse pressure. Thus the mechanical effectiveness of the aortic chamber in receiving the cardiac output and passing it on to the peripheral circulation diminishes progressively with increasing pressures. From this it can readily be understood why the elasticity response of the aorta is physically hampered in cases of hypertension associated with high diastolic pressures.

The effect of diminution in arterial elasticity associated with advancing years is demonstrated by Curve $e$. Even within the range of normal diastolic pressures, the curve clearly shows a comparatively limited degree of elasticity response. It is obvious that the coefficient of elasticity is greatly altered with age. For unit increase in pressure the aorta from younger persons is distended much more than the aorta from individuals of older age groups.

However, the absolute volume of the aorta per unit of length is greater under conditions of low pressure in the older than it is in the younger age groups. Figure 3 illustrates this situation for the eighteen aortas studied completely. Several interesting points are apparent in this graph. The aortas of age group 20 to 24 years (Curve a) show much smaller volumes at $100 \mathrm{~mm}$. $\mathrm{Hg}$ pressure than do those of ages 71 to 78 years (Curve $e$ ). Yet the volumes at higher pressures (200 to $225 \mathrm{~mm}$. $\mathrm{Hg}$ ) are considerably greater in the young than in the old aortas. Thus, the greater extensibility of the younger aorta permits greater dilation under hypertensive conditions than does the older.

The high absolute volume (and diameter) of the older aorta at pressures around $100 \mathrm{~mm} . \mathrm{Hg}$ is obviously fortunate. The work of the heart is less when the diameter of the aorta is great because less work has to be done in giving the blood velocity. The decreased extensibility at higher pressures (for the old as compared with the younger aorta) is apparently an unfavorable change.

The data can be presented in another way by plotting the volume change of the aorta between systolic and diastolic pressure levels. This is a change in aortic pulse volume. If one takes the change occurring between 75 and $125 \mathrm{~mm}$. Hg, 
which is the "normal type" of pulse pressure relation, and also the volume change occurring between 125 and $225 \mathrm{~mm}$. $\mathrm{Hg}$, which approximates a typical " hypertensive" pressure situation tually less under hypertensive conditions than under normal pressure conditions, although the pulse pressure was taken as $100 \mathrm{~mm} . \mathrm{Hg}$, or twice the normal in the hypertensive situation. This is

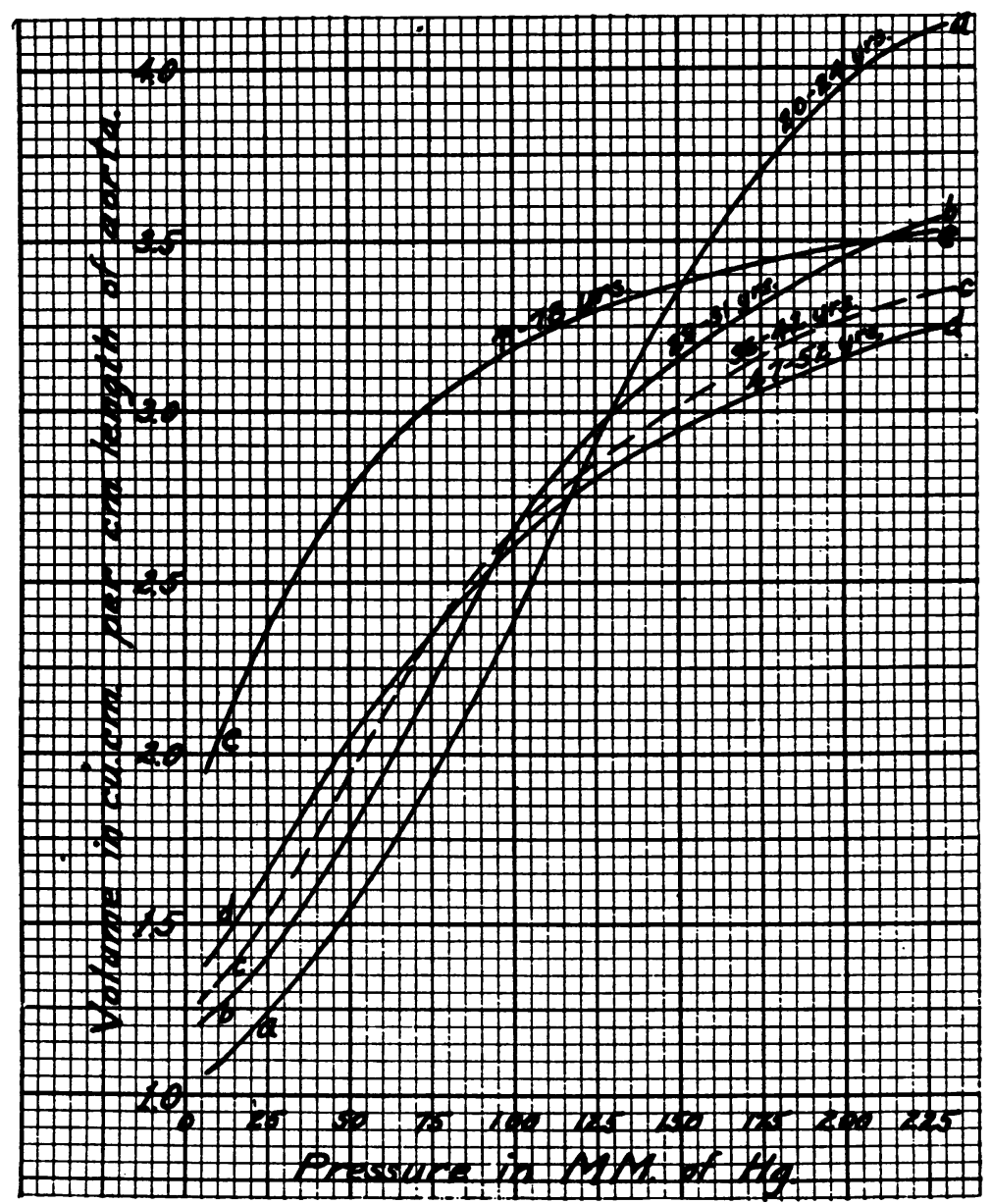

Fig. 3. Curves Showing the Absolute Volume per Unit Length of Aorta at Pressures UP to 230 m M. Hg

It will be noted that the senile aorta has a relatively large volume at normal pressures and that the younger aorta has a greater volume at hypertensive pressures.

in diastole and systole respectively, one can observe certain important facts. Figure 4 presents this data graphically. It can be seen that the "pulse volume change" of the aorta diminishes to 25 per cent or less of that found in the young by the time age 75 has been reached. The aorta no longer acts as an elastic reservoir whose recoil can keep up the pressure and the flow in diastole. Furthermore, and this might not have been predicted, the aortic "pulse volume change" is ac- true because the volume-elasticity curve (see Figure 2) is so nearly flat at the higher pressures in the older aortas. Thus, not only does the aorta become inferior as an elastic reservoir with age, but the hypertension itself makes the aorta function over a range of pressures over which its volume change is less than it would be with pulse pressures half as great at normal systolic pressures. The arteriosclerotic aorta would apparently function better at low pressures. 


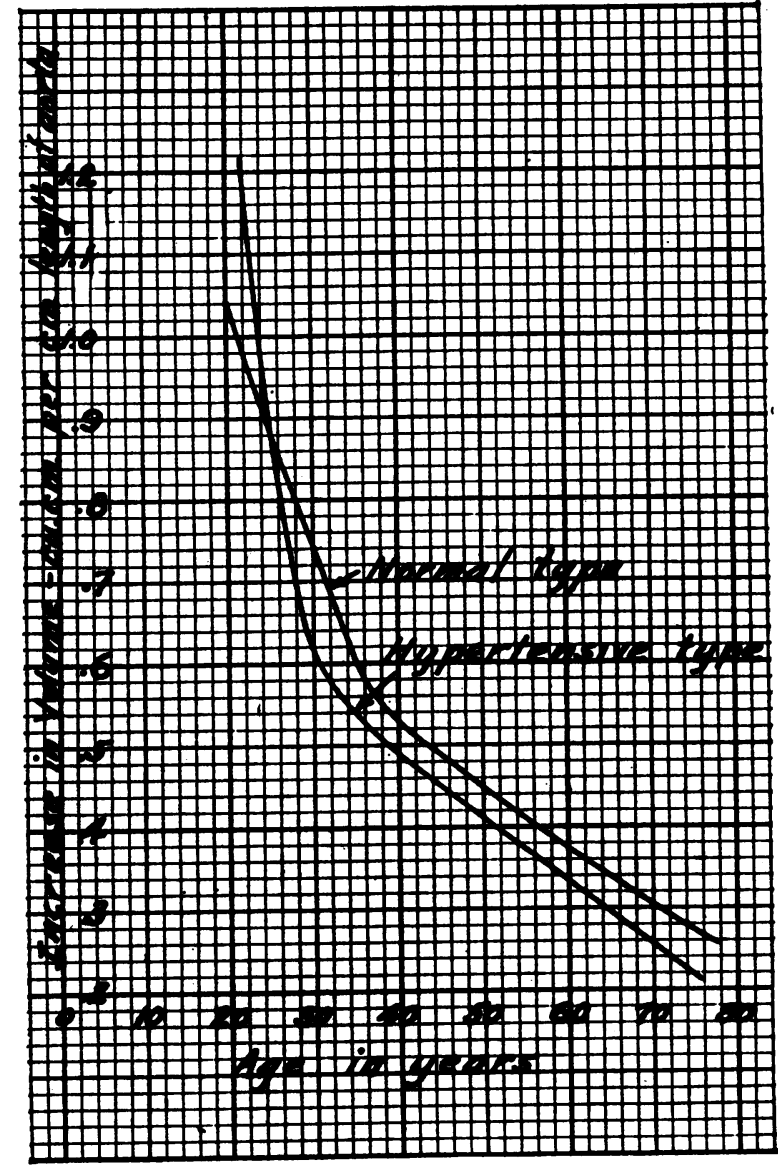

Fig. 4. Curves Showing the Change in Pulse Volume With INCREasing Age for Both Normal aNd Hypertensive Pulse Pressures (50 and 100 m m. Hg RESPECTIVELY)

Anatomical studies of the elastic arteries in the senile period show that the aorta increases in length as well as in diameter. There is a marked lack of uniformity in length and diameter in the aortas of the senile period. A large proportion show marked ectatic dilatation while in others the dilatation is not so pronounced. Suffice it to say that they all show a definite longitudinal as well as a transverse enlargement of various degrees. Thinning of the aortic wall accompanies this alteration. Thus it is evident that the aorta can accommodate a greater volume of blood in its resting state than can the aorta of the younger age groups. What functional capacity the senile aorta loses by way of diminution in its elastic tissue is compensated for to a large extent, by an increase in its size. In other words, the senile aorta becomes essentially a capacity chamber and does not need to be distended in order to accommodate the systolic discharge of the left ventricle without the production of very high velocities of flow. Instead, due to the increased diameter, the stroke output can be carried away at relatively low velocities and therefore with relatively low energy expenditure of the heart.

The above alteration that 'occurs in the aortas of the older age group should be looked upon as a normal, as well as a favorable, physiological adjustment to the ageing process, whereby the deleterious effect of increasing rigidity of the aorta and its main branches upon the heart is prevented. This accounts for the fact that the so-called "uncoiling" of the aorta (elongation and dilatation of this vessel) in the absence of hypertension is, as a rule, associated with hearts of normal size in elderly people $(9,10)$.

\section{A COMPARISON OF THE EXTENSIBILITY OF LIVING AORTA WITH THAT OF ISOLATED AORTA}

As shown by Bramwell and Hill (2), the pulse wave velocity may be derived from the Moen equation and expressed in a convenient formula:

$$
V=\frac{3.57}{\sqrt{\left(\begin{array}{c}
\text { (percentage increase in volume per } \\
\text { mm. Hg increase of pressure })
\end{array}\right.}},
$$

in which $V$ is the velocity of the front of the pulse wave in terms of meters per second. Therefore, it is possible to calculate the velocity of transmission of the pulse wave from the mean volume-elasticity curves for the designated ages and thereby compare the pulse wave velocity of vessels in vivo with those of isolated ones. The pulse wave velocities given below were calculated from the tangents to the respective curves (Figure 2) after being reconstructed on the basis of the normal volumes, assuming a pressure of 85 $\mathrm{mm} . \mathrm{Hg}$. Obviously, the volume at zero pressure cannot be used as a basis for calculation since that volume does not correspond to the volume in vivo. Hence, the normal volume of the aorta at normal internal pressure must be used to calculate the pulse wave velocity since that is the volume which varies in accordance with pressure changes occurring in the aortic chamber in life. 
TABLE I

Calculated and optically-measured pulse wave velocities, in terms of meters per second, and the resulting percentage differences for the various age groups

\begin{tabular}{c|c|c|c|c|c}
\hline \hline $\begin{array}{c}\text { Age } \\
\text { groups }\end{array}$ & $\begin{array}{c}\text { Velocity calcu- } \\
\text { lated from vol- } \\
\text { ume-elasticity } \\
\text { curves of } \\
\text { isolated } \\
\text { aortas }\end{array}$ & $\begin{array}{c}\text { Velocity } \\
\text { in vivo } \\
\text { by } \\
\text { optical } \\
\text { recording } \\
(7) *\end{array}$ & $\begin{array}{c}\text { Velocity in } \\
\text { vivo by } \\
\text { optical } \\
\text { recording } \\
\text { minus 0.4 } \\
\text { meters }\end{array}$ & $\begin{array}{c}\text { Differ- } \\
\text { ence }\end{array}$ & $\begin{array}{c}\text { Percent- } \\
\text { age } \\
\text { differ- } \\
\text { ence }\end{array}$ \\
\hline years & & & & & per cent \\
$20-24$ & 4.3 & 5.2 & 4.8 & 0.5 & 10.4 \\
$29-31$ & 5.0 & 5.6 & 5.2 & 0.2 & 3.9 \\
$36-42$ & 5.8 & 6.4 & 6.0 & 0.2 & 3.3 \\
$47-52$ & 6.5 & 7.5 & 7.1 & 0.6 & 8.4 \\
$71-78$ & 9.5 & 10.5 & 10.1 & 0.6 & 5.9 \\
\hline
\end{tabular}

Average percențage difference $=6.4$ per cent

* These values represent the mean pulse wave velocities of aortas for the various ages as obtained from a study of 550 normal living subjects. See reference 7 of the bibliography.

In Table I, the calculated velocity for each of the age groups is given in the second column. The velocity obtained by the optical recording method (7), and the velocity after subtracting 0.4 meter per second, are given in the third and fourth columns respectively. Obviously, the velocity obtained by the optical recording method includes the velocity of the blood flow itself which is not present nor accounted for in the velocity calculated from the volume-elasticity curves. This velocity of blood flow is approximately 0.4 meter per second (11) in the aorta, which value must be subtracted before comparing with the calculated velocity. The difference between this resulting velocity and the calculated velocity is given in the fifth column, and the percentage difference of the measured velocity is given in the last column. The agreement between the values for the actual pulse wave velocity measured on living persons and that calculated from the volume-elasticity curves is good, namely, 6.4 per cent average percentage difference. Incidentally, the percentage differences with increasing age show a striking constancy (about 6.4 per cent), thus indicating the accuracy as well as the validity of Bramwell and Hill's formula.

Another factor, the effect of elastic "afteraction," tends to produce slower calculated velocities in isolated vessels. By using the described experimental method, this factor is almost but not entirely eliminated. In measuring the volume of the isolated aorta, we found that at the same initial pressures before and after the experiment, the volume after the successive increments of pressure were applied, was increased from 0.75 to 1.0 cubic centimeter or about 8 per cent greater than the original volume, especially in the aortas of older subjects. Since 'the isolated aortas cannot " recoil" to its original or normal diameter, a greater increase in volume would be produced by this method than if completely instantaneous readings could have been made. Hence a 4 per cent slower calculated pulse-wave velocity results. Taking this into consideration and correcting the velocities determined from volume-elasticity curves, the data of Table II is obtained.

TABLE II

Calculated pulse wave velocities corrected for elastic "after-action," optically-measured velocities, in terms of meters per second, and the resulting percentage differences for the various age groups

\begin{tabular}{|c|c|c|c|c|}
\hline $\begin{array}{c}\text { Age } \\
\text { groups }\end{array}$ & $\begin{array}{l}\text { Calculated veloc- } \\
\text { ity corrected for } \\
8 \text { per cent "elastic } \\
\text { after-action" }\end{array}$ & $\begin{array}{l}\text { Mean velocities } \\
\text { measured in } \\
\text { vivo (Table I } \\
\text { corrected for } \\
\text { velocity of } \\
\text { blood flow) }\end{array}$ & $\begin{array}{l}\text { Differ- } \\
\text { ences }\end{array}$ & $\begin{array}{l}\text { Percent- } \\
\text { age } \\
\text { differ- } \\
\text { ence }\end{array}$ \\
\hline $\begin{array}{c}\text { years } \\
20-24 \\
29-31 \\
36-42 \\
47-52 \\
71-78\end{array}$ & $\begin{array}{l}4.5 \\
5.2 \\
6.0 \\
6.8 \\
9.9\end{array}$ & $\begin{array}{r}4.8 \\
5.2 \\
6.0 \\
7.1 \\
10.1\end{array}$ & $\begin{array}{l}0.3 \\
0.0 \\
0.0 \\
0.3 \\
0.2\end{array}$ & $\begin{array}{c}\text { per cent } \\
6.2 \\
0.0 \\
0.0 \\
5.0 \\
2.0\end{array}$ \\
\hline
\end{tabular}

Average percentage difference $=2.6$ per cent

There are several other factors that may account for the 2.6 per cent difference which still exists. It must be remembered that the aorta is surrounded in the living man by a considerable amount of dense fibrous tissue. In all probability this tends to increase the rigidity of the arterial wall somewhat and thus increase the velocity of the pulse wave, whereas in the isolated aortic segment, the layer of dense fibrous tissue was removed in order to ligate the bases of the intercostal vessels. No exact physical measurements are yet available which demonstrate what effect this coating of fibrous tissue has on the extensibility of the aortic chamber. Another factor that may account in part for the abovementioned difference is the actual diastolic pressure present in the aorta. The above tabulated velocities for isolated aortas were computed on a basis of 85 
$\mathrm{mm}$. Hg pressure. It is obviously impossible to obtain the exact diastolic pressure in the living aorta. Undoubtedly it is higher than the normal diastolic pressure as measured over the brachial artery.

Aside from the factors just discussed, it is evident that the velocities of the pulse wave as calculated from volume-elasticity measurements on the isolated aorta are in substantial agreement with that obtained by the optical recording method in vivo.

\section{CONCLUSIONS}

1. A method for experimentally obtaining the volume-elasticity coefficients of isolated aortas has been described.

2. The mean volume-elasticity curves demonstrate that arterial rigidity increases, (a) progressively with age and $(b)$ with increasing diastolic pressure.

3. The aorta of old age assumes the rôle of a capacity chamber or reservoir and by virtue of this readjustment, it becomes adapted to the reception of the cardiac output without imposing an undue strain on the heart, a condition which would otherwise result if the disappearance of elastic tissue occurred without a concomitant increase in the diameter and length of the aorta.

4. If the pulse wave velocities are calculated from mean volume-elasticity curves and compared with the mean pulse wave velocities obtained in living man at corresponding ages, it is found that the mean values obtained on the isolated aorta are less than those obtained in vivo by about 6 per cent. In the study of isolated aortas, the velocity values are slightly low due largely to the factor of elastic " after-action."

5. Due to the satisfactory agreement of the pulse wave velocities found in vivo and in the isolated aortas it is possible to evaluate the condition of the aorta in living man by comparison with an isolated aorta having approximately the same pulse wave velocity.

\section{BIBLIOGRAPHY}

1. Roy, C. S., The elastic properties of the arterial wall. J. Physiol., 1880-82, 3, 125.

2. Bramwell, J. C., and Hill, A. V., The velocity of the pulse wave in man. Proc. Roy. Soc., ser. B, 1922, 93, 298.

3. Bramwell, J. C., Downing, A. C., and Hill, A. V., The effect of blood pressure on the extensibility of the human artery. Heart, 1923, 10, 289.

4. Zon, L., Elasticity of the aorta. Unpublished paper. University of Minnesota Thesis. 1932.

5. Bramwell, J. C., From Cowdry, E. V., Arteriosclerosis; A Survey of the Problem. Macmillan Co., New York, 1933, pp. 77 to 119.

6. Aschoff, L., From Cowdry, E. V., Arteriosclerosis ; A Survey of the Problem. Macmillan Co., New York, 1933, p. 4.

7. Hallock, P., Arterial elasticity in man in relation to age as evaluated by the pulse wave velocity method. Arch. Int. Med., 1934, 54, 770.

8. Moens, Die Pulscurve. E. J. Brill, Leiden, 1878.

9. Brodin, P., Heim de Balsac, R., and Tedesco, Etude Radiologique de l'appareil cardio-vasculaire des vieillards. Gaz. méd. de France (Supp. Radiol.), Feb. 15, 1935, p. 310.

10. Parkinson, J., Enlargement of the heart. Lancet, 1936, 1, 1337.

11. Evans, C. L., The velocity factor in cardiac work. J. Physiol., 1918-19, 52, 6. 